OPEN ACCESS

Edited by:

Vincent Tropepe,

University of Toronto, Canada

Reviewed by:

Matteo Bergami,

Cologne Excellence Cluster on Cellular

Stress Responses in Aging

Associated Diseases, Germany

Carmen Vivar,

Centro de Investigación y de Estudios Avanzados del Instituto Politécnico Nacional (CINVESTAV-IPN), Mexico

${ }^{*}$ Correspondence:

Patrick Z. Liu

patrick.liu@northwestern.edu

Specialty section: This article was submitted to

Neurogenesis,

a section of the journal

Frontiers in Neuroscience

Received: 19 November 2017

Accepted: 23 January 2018

Published: 07 February 2018

Citation:

Liu PZ and Nusslock R (2018) Exercise-Mediated Neurogenesis in

the Hippocampus via BDNF.

Front. Neurosci. 12:52.

doi: 10.3389/fnins.2018.00052

\section{Exercise-Mediated Neurogenesis in the Hippocampus via BDNF}

\author{
Patrick Z. Liu* and Robin Nusslock \\ Department of Psychology, Northwestern University, Evanston, IL, United States
}

Exercise is known to have numerous neuroprotective and cognitive benefits, especially pertaining to memory and learning related processes. One potential link connecting them is exercise-mediated hippocampal neurogenesis, in which new neurons are generated and incorporated into hippocampal circuits. The present review synthesizes the extant literature detailing the relationship between exercise and hippocampal neurogenesis, and identifies a key molecule mediating this process, brain-derived neurotrophic factor (BDNF). As a member of the neurotrophin family, BDNF regulates many of the processes within neurogenesis, such as differentiation and survival. Although much more is known about the direct role that exercise and BDNF have on hippocampal neurogenesis in rodents, their corresponding cognitive benefits in humans will also be discussed. Specifically, what is known about exercise-mediated hippocampal neurogenesis will be presented as it relates to BDNF to highlight the critical role that it plays. Due to the inaccessibility of the human brain, much less is known about the role BDNF plays in human hippocampal neurogenesis. Limitations and future areas of research with regards to human neurogenesis will thus be discussed, including indirect measures of neurogenesis and single nucleotide polymorphisms within the BDNF gene.

Keywords: neurotrophin, TrkB, subgranular zone, neuroprotective, dentate gyrus

\section{INTRODUCTION}

For centuries, researchers have sought to elucidate the mechanisms behind the axiom that a healthy body leads to a healthy mind. It has now been established that exercise, even among minimal commitment exercise routines, has an array of robust effects on the brain, such as enhanced memory, mood, cognitive functioning, plasticity, and learning capabilities (Erickson et al., 2011; Spalding et al., 2013; Phillips et al., 2014). Most notably, exercise has been implicated in having anti-depressant effects and counteracting disease or age-related mental impairment and atrophy, such as Alzheimer's disease or dementia (Laurin et al., 2001). Yet, until recently, the intermediaries between exercise and its health benefits have not been well-understood.

However, it has been shown that-contrary to the age-old notion that the number of neurons in the brain remains static after prenatal and neonatal development-new neurons can be generated in the adult brain via a process known as neurogenesis, which can attenuate the deleterious effects of neurodegeneration (van Praag et al., 1999). This phenomenon has been linked to exercise, with a significant portion of subsequent neural growth occurring in the dentate gyrus of the hippocampus (Cotman and Berchtold, 2002). Since the hippocampus is critical for memory consolidation and learning, the generation of new neurons and increased plasticity in this brain region may explain the improved cognition and emotional state that accompanies exercise (Gandy et al., 2017; Trinchero et al., 2017). Furthermore, preliminary research has suggested that neurogenesis may also occur in numerous other areas of the brain, including the amygdala and hypothalamus, which may 
explain the diversity of exercise-derived benefits (Fowler et al., 2008). However, this research is not as extensive or conclusive as hippocampal neurogenesis research, nor is the extent to which neurogenesis occurs in other brain regions as robust as it is in the hippocampus, with the exception of the olfactory bulb (Cotman et al., 2007).

As such, this review will exclusively summarize the effects of exercise on neurogenesis in the hippocampus. This paper will also explicate a key molecule, brain-derived neurotrophic factor (BDNF), that has been shown to modulate neurogenesis and how exercise influences BDNF levels. Lastly, due to discrepancies between rodent and human studies, limitations and future areas of investigation with regards to neurogenesis in humans will be discussed.

\section{EXERCISE AND HIPPOCAMPAL NEUROGENESIS}

In rodents, hippocampal neurogenesis as a function of exercise has been extensively demonstrated and replicated. To test this, rodents are injected with bromodeoxyuridine (BrdU), which signify actively mitotic cells and are incorporated by daughter cells, thereby allowing the tracing of cell division (del Rio and Soriano, 1989). In some of the earliest work in this field, it was shown that mice allowed to voluntarily exercise on a running wheel exhibited enhanced neurogenesis in the dentate gyrus. By utilizing BrdU as a tracing molecule, it was observed that exercise not only increased proliferation of the progenitor cells in the subgranular zone, but also increased their survival rate as they differentiated and matured (van Praag et al., 1999; Seri et al., 2001; for reviews of neural progenitors and lineage progression see Weissman et al., 2001; Seri et al., 2004; Göritz and Frisén, 2012).

Although it is much more difficult to study exercise-mediated neurogenesis in humans, there is significant evidence that neurogenesis occurs in the adult human brain, especially in the dentate gyrus. Indeed, exercise has been shown to increase the size of the hippocampus in human adults (Erickson et al., 2011). Through postmortem tissue analysis of cancer patients administered BrdU, it has been shown that mature granule neurons are continually generated from the subgranular zone, even in the later stages of life (Eriksson et al., 1998). Interestingly, the participants in this study were not assigned to exercise conditions, and since they were cancer patients near death, it is unlikely they participated in any exercise regimen. This suggests that the hippocampus has the capability to generate new neurons in adulthood independent of exercise. Later sections in this review, however, provide evidence that exercise accentuates neurogenesis in humans and addresses how the amount of exercise modulates the degree of neurogenesis.

\section{BDNF MEDIATION OF HIPPOCAMPAL NEUROGENESIS}

Given these early findings establishing a connection between exercise and hippocampal neurogenesis, researchers next turned to elucidating the biological underpinnings. One of the strongest candidates for bridging the gap between exercise and neurogenesis is BDNF, a growth factor categorized under the neurotrophin family widely expressed in the brain and throughout the rest of the central nervous system (Salehi et al., 2003). Early research on this molecule found that during development in mice, BDNF expression is low during prenatal development, but then increases during the first few weeks after being born and peaks during the shift from embryonic to adult neurogenesis (Bath et al., 2012). This provides key insight into its potential for facilitating neurogenesis, which then spurred much more research interest in its connection to neurogenesis.

\section{BDNF}

As a whole, the neurotrophin family polypeptides are vital to the regulation of the neural processes in neurogenesis, such as proliferation, differentiation, maturation, and plasticity. Within this family, BDNF exhibits the highest degree of expression in the brain, and is primarily synthesized there during exercise (Reichardt, 2006). BDNF can also enter the brain via freely diffusing across the blood brain barrier (Pan et al., 1998; Mousavi and Jasmin, 2006). Furthermore, during exercise, proteins and their metabolic derivatives secreted from peripheral muscles, such as cathepsin B and FNDC5/irisin, also cross the blood brain barrier to mediate BDNF expression in the hippocampus and subsequent neurogenesis and memory improvement (Wrann et al., 2013; Moon et al., 2016). Indeed, mice injected with skeletal muscle endurance factors had elevated levels of hippocampal neurogenesis and increased spatial memory (Kobilo et al., 2010).

BDNF functions by binding to tropomyosin receptor kinase B (TrkB), which is largely expressed in hippocampal neurons. Upon binding, the BDNF-TrkB complex is then internalized into the neuron and serves as a docking site for numerous signaling cascades, protein phosphorylation cascades, and secondary signaling systems (Huang and Reichardt, 2003; Nykjaer et al., 2005; Yoshii and Constantine-Paton, 2010; for review of signaling pathways see Phillips et al., 2014). Through these pathways, BDNF can exert significant regulatory control over many facets of a neuron's function and thereby influence how these neurons function as a whole within the hippocampus.

\section{BDNF and TrkB Influences on the Hippocampus}

Given the vast array of possible signaling pathways through which BDNF can operate, it is not surprising that BDNF expression impacts multiple aspects of the hippocampus, including cognitive functioning and neurogenesis. In terms of cognitive processes, higher levels of BDNF expression have been implicated in long-term potentiation of neurons and synaptic plasticity, which may explain why higher levels of BDNF expression are associated with enhanced spatial and verbal memory and recognition capabilities, and may also counteract the effects of chronic stress and cognitive decline (Korte et al., 1995; Voss et al., 2013b; Wang et al., 2015; Liu and Nusslock, 2018; for reviews of plasticity see Lu et al., 2013, 2014; Karpova, 2014; Leal et al., 2015). Indeed, in mice, $\mathrm{BDNF}$ is required for hippocampal neurogenesis spurred by 
an enriched environment, and BDNF has been shown to have coordinated effects with antidepressants on neuronal progenitor cells in the hippocampus (Sairanen et al., 2005; Rossi et al., 2006). Furthermore, decreased BDNF levels, especially in older adults, may lead to compromised memory, neurodegeneration, and various other cognitive impairments found in Alzheimer's disease (for review, see Zuccato and Cattaneo, 2009).

To study BDNF's role in neurogenesis, researchers have utilized BDNF and TrkB knockdown mice and also exogenous factors to overexpress BDNF. In BDNF knockdown mice, it was observed that the neural stem cells in the subgranular zone proliferated significantly less than in wild type mice. Moreover, significantly more of the stem cells that did proliferate died in the BDNF knockdown mice before fully differentiating and maturing (Lee et al., 2002). These results suggest that at the very least, BDNF is necessary for a basal level of neurogenesis. Further work corroborated these findings by demonstrating that in TrkB knockdown mice, both proliferation and differentiation of neural stem cells are significantly reduced, thereby suggesting that BDNF and TrkB are functionally dependent upon each other and that altering TrkB responsivity affects neurogenesis equally as altering BDNF expression levels (Bartkowska et al., 2007; Li et al., 2008). Removal of TrkB also stunted dendritic growth and adult progenitor integration into the hippocampus in adult mice, which led to more pronounced anxious behavior (Bergami et al., 2008).

However, when BDNF expression levels were exogenously increased in mice with wild type $\operatorname{TrkB}$, these effects were reversed and even accentuated. Even when administered a single injection of BDNF, substantially more neurons were recruited from neuronal progenitor cells (Benraiss et al., 2001). And when chronically stimulated with BDNF expression, a significant increase in net neurogenesis was observed in the dentate gyrus (Quesseveur et al., 2013). In fact, many individual studies have found that increased BDNF expression robustly promotes the in vivo proliferation, triggering of differentiation, axonal path migration, and maturation of the neural stem cells in the dentate gyrus (Korte et al., 1995; Reichardt, 2006; Nakata and Nakamura, 2007; Waterhouse et al., 2012). Through an autocrine loop initiated by BDNF, these newly generated neurons are also better protected against cell death (Acheson et al., 1995).

Therefore, considering both the knockdown and overexpression studies in tandem, there is an established connection between BDNF and neurogenesis. Since BDNF also promotes neuronal survival and enhanced nerve transmission via long-term potentiation, this combination of neurogenesis and optimized neuronal functioning significantly improves cognitive performance and protects against neurodegenerative phenomena.

\section{Exercise and BDNF Expression}

Thus far, both exercise and BDNF have been shown to be associated with increased neurogenesis. Further research has extended this to show that treadmill exercise in mice and aerobic exercise in humans increases BDNF expression by regulating BDNF gene expression in the hippocampus (Kim et al., 2015). This process is largely mediated by neurotransmitter and neuroendocrine systems, with extensive literature supporting acetylcholine (ACh) as a key regulator (Knipper et al., 1994).

In mice allowed to voluntarily engage in wheel running, an increase in BDNF mRNA levels in the dentate gyrus was observed after only a few days of exercise (Neeper et al., 1995). Surprisingly, these levels were maintained throughout several weeks of exercise and corresponded to proportional increases in BDNF protein expression (Russo-Neustadt et al., 1999). When the exercise conditions were supplemented with antibodies blocking TrkB, however, the mice had attenuated learning capabilities involving the hippocampus. Furthermore, these mice also lacked synapticspecific proteins in the hippocampus, thereby demonstrating that BDNF signaling is necessary to allow the benefits of exercise to manifest (Vaynman et al., 2004, 2006).

Research has also shown that metrics of overall health quality in humans follow a dose-dependent relationship with the duration and intensity of exercise, with the best outcomes linked to moderate exercise (Larson et al., 2006). Further work illustrates that mice show greater improvements in acquisition and retention based learning in hippocampus-dependent tasks following long-term exercise rather than shorter regimes of exercise (Handschin and Spiegelman, 2008; Parachikova et al., 2008; Ploeger et al., 2009). It was found that in mice, even just one session of exercise increased BDNF levels. This effect, however, became amplified following a period of exercise in mice that regularly exercised, with an increased response in BDNF levels relative to mice after just a single session of exercise (Johnson et al., 2003; van Praag et al., 2005; Rasmussen et al., 2009). Consistent with these findings is a meta-analysis of 29 studies spanning 1,111 human participants that analyzed BDNF expression levels across various exercise paradigms. However, many of the studies only examined moderate exercise, and several studies did not report intensity level. Interestingly, considerable evidence from this meta-analysis suggests that humans also experience a dose-response relationship in which each session of exercise corresponds to a dose of increased BDNF expression. Furthermore, regular exercise in moderate amounts has been shown to increase the magnitude of BDNF expression following individual sessions of exercise (Szuhany et al., 2015).

There is not a perfect positive correlation, however, between the amount and intensity of exercise and BDNF expression levels and subsequent health benefits. Extreme exercise has been shown to disrupt a number of metabolic and physiological processes and lead to impaired cognitive performance in humans (Aguiló et al., 2005). Since oxygen is rapidly metabolized during physical exertion, reactive oxygen species (ROS) are naturally produced as a metabolic byproduct. When produced at high levels, such as during bouts of intense exercise, ROS can lead to oxidative damage and increased cellular mortality in both rodents and humans (Radak et al., 2016). Moderate levels of exercise enforce the human body's antioxidant defense system, but extreme levels of exercise lead to the generation of more ROS than the antioxidant system can defend against, thereby allowing their accumulation as oxidative stress (Mastaloudis et al., 2001). In fact, when treated with hydrogen peroxide, a potent ROS, hippocampal cell cultures taken from rodents showed an inverse relationship between BDNF expression levels 
and hydrogen peroxide concentration (Kwon et al., 2013). The in vivo production of BDNF as a function of ROS production, however, is less clear and warrants further study.

\section{LIMITATIONS AND FUTURE DIRECTIONS}

Both optimized health outcomes and increased BDNF levels have been shown to follow moderate exercise in humans. Given how these results are consistent in mice studies and how BDNF is a highly conserved growth factor across numerous species, it is likely that BDNF also heavily mediates exercise-induced neurogenesis in the dentate gyrus in humans. However, due to ethical reasons, it is difficult to conclusively test this since brain samples cannot be readily collected from human participants following various exercise paradigms.

Furthermore, even if neurogenesis could be tested in humans following different exercise routines, it would be nearly impossible to track the time course of new neuronal development since this would require harvesting brain tissue postmortem. As such, neurogenesis studies in humans need to rely on indirect measures, such as administering cognitive tests related to memory consolidation or measuring neurobiological indicators of neurogenesis and plasticity, such as BDNF (Voss et al., 2013a). A potential indirect measurement that has been established is the correlation between cerebral blood volume in the dentate gyrus, cognitive enhancements, and adult neurogenesis as a function of exercise (Pereira et al., 2007). Although this correlation is robustly established in mice, it is still difficult to conclusively connect cerebral blood volume to neurogenesis in humans. Accompanying studies in rodents should therefore definitively establish how indicative these indirect measures are of neurogenesis and also seek to determine causality so that human neurogenesis can be better assessed and analyzed following exercise or BDNF supplementation. Although discrepancies between animal model and human studies exist (Spalding et al., 2013; Paredes et al., 2016), the outlook for future studies should be optimistic since a combined approach of analyzing BDNF levels, cognitive functioning, and cerebral blood volume could provide significant evidence suggesting adult neurogenesis.

Another factor to consider in this field of research is the contribution of gene-environment interactions. The most heavily studied single nucleotide polymorphism (SNP) in the BDNF gene is rs6265, otherwise known as val66met. This SNP reduces BDNF's distribution within neurons and also its protein binding affinity, which has been linked to neuropathology and cognitive deficiencies (Chiaruttini et al., 2009). In a meta-analysis of 399 healthy participants, met carriers were found to have significantly decreased hippocampal volumes (Hajek et al., 2012). In an extensive longitudinal study, the presence of rs6265 robustly

\section{REFERENCES}

Acheson, A., Conover, J. C., Fandl, J. P., DeChiara, T. M., Russell, M., Thadani, A., et al. (1995). A BDNF autocrine loop in adult sensory neurons prevents cell death. Nature 374, 450-453. doi: 10.1038/374450a0 predicted the gradual decline of hippocampal volume and also subsequent skilled task performance (Sanchez et al., 2011). This SNP has also been associated with the occurrence of major depressive disorder, anxiety disorders, suicidal tendencies, and depressive episodes in bipolar disorder and in patients with Alzheimer's disease (Kim et al., 2008; Terracciano et al., 2010).

Interestingly, there appears to be a bidirectional relationship between various BDNF polymorphisms and the environment that future studies should account for. The presence of a val2met substitution has been shown to hinder working memory and other aspects of cognition, yet high levels of exercise counteracted these cognitive impairments, suggesting that exercise may be able to modulate genetic influences (Erickson et al., 2013). However, in other forms of BDNF polymorphisms, adenine alleles in the polymorphisms have been shown to decrease compliance in participants to engage in highly demanding exercise regimens (Bryan et al., 2013). As such, the interaction of BDNF polymorphisms with exercise is difficult to disambiguate and needs further elucidation.

Lastly, another area for further study will be to directly measure BDNF expression as a function of exercise intensity, duration, and sedentary status. Although many studies mentioned in this review analyzed BDNF expression from moderate exercise, much less is known about how different levels of exercise intensity directly affect BDNF levels and subsequent neurogenesis. Additionally, in the human studies, a person's sedentary status described as sedentary, regular exercise, or athlete was either not noted or controlled for via randomization studies. As such, it is also unclear how sedentary status combined with exercise intensity can modulate BDNF expression (Szuhany et al., 2015).

Despite what is still yet unknown regarding exercise-mediated neurogenesis and BDNF, it is nonetheless known that exercise has immense benefits for physical, psychological, developmental, and social factors. Exercise, when pursued in moderation, not only serves as a robust method for improving physical health, but also serves as a preventative and protective measure against numerous neurological and mental diseases. Neurogenesis is still a poorly understood topic, and as the role and benefits of exercise are continually being better elucidated, future research initiatives on exercise will undoubtedly uncover more benefits on a personal and social level.

\section{AUTHOR CONTRIBUTIONS}

All authors listed have made a substantial, direct and intellectual contribution to the work, and approved it for publication.

\section{FUNDING}

RN is supported by grants MH100117 and MH077908.

Aguiló, A., Tauler, P., Fuentespina, E., Tur, J. A., Cordova, A., and Pons, A. (2005). Antioxidant response to oxidative stress induced by exhaustive exercise. Physiol. Behav. 84, 1-7. doi: 10.1016/j.physbeh.2004.07.034

Bartkowska, K., Paquin, A., Gauthier, A. S., Kaplan, D. R., and Miller, F. D. (2007). Trk signaling regulates neural precursor cell proliferation and 
differentiation during cortical development. Development 134, 4369-4380. doi: $10.1242 / \mathrm{dev} .008227$

Bath, K. G., Akins, M. R., and Lee, F. S. (2012). BDNF control of adult SVZ neurogenesis. Dev. Psychobiol. 54, 578-589. doi: 10.1002/dev.20546

Benraiss, A., Chmielnicki, E., Lerner, K., Roh, D., and Goldman, S. A. (2001), Adenoviral brain-derived neurotrophic factor induces both neostriatal and olfactory neuronal recruitment from endogenous progenitor cells in the adult forebrain. J. Neurosci. 21, 6718-6731.

Bergami, M., Rimondini, R., Santi, S., Blum, R., Götz, M., and Canossa, M. (2008). Deletion of TrkB in adult progenitors alters newborn neuron integration into hippocampal circuits and increases anxiety-like behavior. Proc. Natl. Acad. Sci. U.S.A. 105, 15570-15575. doi: 10.1073/pnas.0803702105

Bryan, A. D., Magnan, R. E., Hooper, A. E., Ciccolo, J. T., Marcus, B., and Hutchison, K. E. (2013). Colorado stride (COSTRIDE): testing genetic and physiological moderators of response to an intervention to increase physical activity. Int. J. Behav. Nutr. Phys. Acta 10:139. doi: 10.1186/1479-5868-10-139

Chiaruttini, C., Vicario, A., Li, Z., Baj, G., Braiuca, P., Wu, Y., et al. (2009). Dendritic trafficking of BDNF mRNA is mediated by translin and blocked by the G196A (Val66Met) mutation. Proc. Natl. Acad. Sci. U.S.A. 106, 16481-16486. doi: 10.1073/pnas.0902833106

Cotman, C. W., and Berchtold, N. C. (2002). Exercise: a behavioral intervention to enhance brain health and plasticity. Trends Neurosci. 25, 295-301. doi: 10.1016/S0166-2236(02)02143-4

Cotman, C. W., Berchtold, N. C., and Christie, L. (2007). Exercise builds brain health: key roles of growth factor cascades and inflammation. Trends Neurosci. 30, 464-472. doi: 10.1016/j.tins.2007.06.011

del Rio, J. A., and Soriano, E. (1989). Immunocytochemical detection of 5'bromodeoxyuridine incorporation in the central nervous system of the mouse. Dev. Brain Res. 49, 311-317.

Erickson, K. I., Banducci, S. E., Weinstein, A. M., MacDonald, A. W. III, Ferrell, R. E., Halder, I., et al. (2013). The brain-derived neurotrophic factor val66met polymorphism moderates an effect of physical activity on working memory performance. Psychol. Sci. 24, 1770-1779. doi: 10.1177/09567976134 80367

Erickson, K. I., Voss, M. W., Prakash, R. S., Basak, C., Szabo, A., Chaddock, L., et al. (2011). Exercise training increases size of hippocampus and improves memory. Proc. Natl. Acad. Sci. U.S.A. 108, 3017-3022. doi: 10.1073/pnas.1015950108

Eriksson, P. S., Perfilieva, E., Björk-Eriksson, T., Alborn, A., Nordberg, C., Peterson, D. A., et al. (1998). Neurogenesis in the adult human hippocampus. Nat. Med. 4, 1313-1317. doi: 10.1038/3305

Fowler, C. D., Liu, Y., and Wang, Z. (2008). Estrogen and adult neurogenesis in the amygdala and hypothalamus. Brain Res. Rev. 57, 342-351. doi: 10.1016/j.brainresrev.2007.06.011

Gandy, K., Kim, S., Sharp, C., Dindo, L., Maletic-Savatic, M., and Calarge, C. (2017). Pattern separation: a potential marker of impaired hippocampal adult neurogenesis in major depressive disorder. Front. Neurosci. 11:571. doi: $10.3389 /$ fnins.2017.00571

Göritz, C., and Frisén, J. (2012). Neural stem cells and neurogenesis in the adult. Cell Stem Cell 10, 657-659. doi: 10.1016/j.stem.2012.04.005

Hajek, T., Kopecek, M., and Höschl, C. (2012). Reduced hippocampal volumes in healthy carriers of brain-derived neurotrophic factor Val66Met polymorphism: meta-analysis. World J. Biol. Psychiatry 13, 178-187. doi: 10.3109/15622975.2011.580005

Handschin, C., and Spiegelman, B. M. (2008). The role of exercise and PGC1alpha in inflammation and chronic disease. Nature 454, 463-469. doi: $10.1038 /$ nature 07206

Huang, E. J., and Reichardt, L. F. (2003). Trk receptors: roles in neuronal signal transduction. Annu. Rev. Biochem. 72, 609-642. doi: 10.1146/annurev.biochem.72.121801.161629

Johnson, R. A., Rhodes, J. S., Jeffrey, S. L., Garland, T. Jr., and Mitchell, G. S. (2003). Hippocampal brain-derived neurotrophic factor but not neurotrophin3 increases more in mice selected for increased voluntary wheel running. Neuroscience 121, 1-7. doi: 10.1016/S0306-4522(03)00422-6

Karpova, N. N. (2014). Role of BDNF epigenetics in activitydependent neuronal plasticity. Neuropharmacology 76, 709-718. doi: 10.1016/j.neuropharm.2013.04.002

Kim, J. M., Stewart, R., Kim, S. W., Yang, S. J., Shin, I. S., Kim, Y. H., et al. (2008). BDNF genotype potentially modifying the association between incident stroke and depression. Neurobiol. Aging 29, 789-792. doi: 10.1016/j.neurobiolaging.2006.11.021

Kim, K., Sung, Y., Seo, J., Lee, S., Lim, B., Lee, C., et al. (2015). Effects of treadmill exercise-intensity on short-term memory in the rats born of the lipopolysaccharide-exposed maternal rats. J. Exerc. Rehabil. 11, 296-302. doi: 10.12965/jer.150264

Knipper, M., da Penha Berzaghi, M., Blöchl, A., Breer, H., Thoenen, H., and Lindholm, D. (1994). Positive feedback between acetylcholine and the neurotrophins nerve growth factor and brain-derived neurotrophic factor in the rat hippocampus. Eur. J. Neurosci. 6, 668-671. doi: 10.1111/j.1460-9568.1994.tb00312.x

Kobilo, T., Yuan, C., and van Praag, H. (2010). Endurance factors improve hippocampal neurogenesis and spatial memory in mice. Learn. Mem. 18, 103-107. doi: 10.1101//m.2001611

Korte, M., Carroll, P., Wolf, E., Brem, G., Thoenen, H., and Bonhoeffer, T. (1995). Hippocampal long-term potentiation is impaired in mice lacking brainderived neurotrophic factor. Proc. Natl. Acad. Sci. U.S.A. 92, 8856-8860. doi: $10.1073 /$ pnas.92.19.8856

Kwon, D. H., Kim, B. S., Chang, H., Kim, Y. I., Jo, S. A., and Leem, Y. H. (2013). Exercise ameliorates cognition impairment due to restraint stress-induced oxidative insult and reduced BDNF level. Biochem. Biophys. Res. Commun. 434, 245-251. doi: 10.1016/j.bbrc.2013.02.111

Larson, E. B., Wang, L., Bowen, J. D., McCormick, W. C., Teri, L., Crane, P., et al. (2006). Exercise is associated with reduced risk factor for incident dementia among persons 65 years of age and older. Ann. Intern. Med. 144, 73-81. doi: 10.7326/0003-4819-144-2-200601170-00004

Laurin, D., Verreault, R., Lindsay, J., MacPherson, K., and Rockwood, K. (2001). Physical activity and risk of cognitive impairment and dementia in elderly persons. Archiv. Neurol. 58, 498-504. doi: 10.1001/archneur.58.3.498

Leal, G., Alfonso, P. M., Salazar, I. L., and Duarte, C. B. (2015). Regulation of hippocampal synaptic plasticity by BDNF. Brain Res. 1621, 82-101. doi: 10.1016/j.brainres.2014.10.019

Lee, J., Duan, W., and Mattson, M. P. (2002). Evidence that brainderived neurotrophic factor is required for basal neurogenesis and mediates, in part, the enhancement of neurogenesis by dietary restriction in the hippocampus of adult mice. J. Neurochem. 82, 1367-1375. doi: 10.1046/j.1471-4159.2002.01085.x

Li, Y., Luikart, B. W., Birnbaum, S., Chen, J., Kwon, C., Kernie, S. G., et al. (2008). TrkB regulates hippocampal neurogenesis and governs sensitivity to antidepressive treatment. Neuron 59, 399-412. doi: 10.1016/j.neuron.2008.06.023

Liu, P. Z., and Nusslock, R. (2018). How stress gets under the skin: early life adversity and glucocorticoid receptor epigenetic regulation. Curr. Genomics. doi: 10.2174/1389202919666171228164350. [Epub ahead of print].

Lu, B., Nagappan, G., Guan, X., Nathan, P. J., and Wren, P. (2013). BDNF-based synaptic repair as a disease-modifying strategy for neurodegenerative diseases. Nat. Rev. Neurosci. 14, 401-416. doi: 10.1038/nrn3505

Lu, B., Nagappan, G., and Lu, Y. (2014). BDNF and synaptic plasticity, cognitive function, and dysfunction. Handb. Exp. Pharmacol. 220, 223-250. doi: 10.1007/978-3-642-45106-5_9

Mastaloudis, A., Leonard, S. W., and Traber, M. G. (2001). Oxidative stress in athletes during extreme endurance exercise. Free Radic. Biol. Med. 31, 911-922. doi: 10.1016/S0891-5849(01)00667-0

Moon, H. Y., Becke, A., Berron, D., Becker, B., Sah, N., Benoni, G., et al. (2016). Running-induced systemic cathepsin B secretion is associated with memory function. Cell Metab. 24, 332-340. doi: 10.1016/j.cmet.2016.05.025

Mousavi, K., and Jasmin, B. J. (2006). BDNF is expressed in skeletal muscle satellite cells and inhibits myogenic differentiation. J. Neurosci. 26, 5739-5749. doi: 10.1523/JNEUROSCI.5398-05.2006

Nakata, H., and Nakamura, S. (2007). Brain-derived neurotrophic factor regulates AMPA receptor trafficking to post-synaptic densities via IP3R and TRPC calcium signaling. FEBS Lett. 581, 2047-2054. doi: 10.1016/j.febslet.2007.04.041

Neeper, S. A., Gómez-Pinilla, F., Chol, J., and Cotman, C. (1995). Exercise and brain neurotrophins. Nature 373:109. doi: 10.1038/373109a0

Nykjaer, A., Willnow, T. E., and Petersen, C. M. (2005). P75NTR-live or let die. Curr. Opin. Neurobiol. 15, 49-57. doi: 10.1016/j.conb.2005.01.004

Pan, W., Banks, W. A., Fasold, M. B., Bluth, J., and Kastin, A. J. (1998). Transport of brain-derived neurotrophic factor across the blood-brain 
barrier. Neuropharmacology 37, 1553-1561. doi: 10.1016/S0028-3908(98) 00141-5

Parachikova, A., Nichol, K. E., and Cotman, C. W. (2008). Short-term exercise in aged Tg2576 mice alters neuroinflammation and improves cognition. Neurobiol. Dis. 30, 121-129. doi: 10.1016/j.nbd.2007.12.008

Paredes, M. F., Sorrells, S. F., Garcia-Verdugo, J. M., and Alvarez-Buylla, A. (2016). Brain size and limits to adult neurogenesis. J. Comp. Neurol. 524, 646-664. doi: $10.1002 / \mathrm{cne} .23896$

Pereira, A. C., Huddleston, D. E., Brickman, A. M., Sosunov, A. A., Hen, R., McKhann, G. M., et al. (2007). An in vivo correlate of exercise-induced neurogenesis in the adult dentate gyrus. Proc. Natl. Acad. Sci. U.S.A. 104, 5638-5643. doi: 10.1073/pnas.0611721104

Phillips, C., Baktir, M. A., Srivatsan, M., and Salehi, A. (2014). Neuroprotective effects of physical activity on the brain: a closer look at trophic factor signaling. Front. Cell. Neurosci. 8:170. doi: 10.3389/fncel.2014.00170

Ploeger, H. E., Takken, T., de Greef, M. H., and Timmons, B. W. (2009). The effects of acute and chronic exercise on inflammatory markers in children and adults with a chronic inflammatory disease: a systematic review. Exerc. Immunol. Rev. $15,6-41$.

Quesseveur, G., David, D. J., Gaillard, M. C., Pla, P., Wu, M. V., Nguyen, H. T., et al. (2013). BDNF overexpression in mouse hippocampal astrocytes promotes local neurogenesis and elicits anxiolytic-like activities. Transl. Psychiatry 3:e253. doi: $10.1038 /$ tp. 2013.30

Radak, Z., Suzuki, K., Higuchi, M., Balogh, L., Boldogh, I., and Koltai, E. (2016). Physical exercise, reactive oxygen species and neuroprotection. Free Rad. Biol. Med. 98, 187-196. doi: 10.1016/j.freeradbiomed.2016.01.024

Rasmussen, P., Brassard, P., Adser, H., Pedersen, M. V., Leick, L., Hart, E., et al. (2009). Evidence for a release of brain-derived neurotrophic factor from the brain during exercise. Exp. Physiol. 94, 1062-1069. doi: 10.1113/expphysiol.2009.048512

Reichardt, L. F. (2006). Neurotrophin-regulated signaling pathways. Philos. Trans. R. Soc. B 361, 1545-1564. doi: 10.1098/rstb.2006.1894

Rossi, C., Angelucci, A., Costantin, L., Braschi, C., Mazzantini, M., Babbini, F., et al. (2006). Brain-derived neurotrophic factor (BDNF) is required for the enhancement of hippocampal neurogenesis following environmental enrichment. Eur. J. Neurosci. 24, 1850-1856. doi: 10.1111/j.1460-9568.2006.05059.x

Russo-Neustadt, A., Beard, R. C., and Cotman, C. W. (1999). Exercise, antidepressant medications, and enhanced brain derived neurotrophic factor expression. Neuropsychopharmacology 21, 679-682. doi: 10.1016/S0893-133X(99)00059-7

Sairanen, M., Lucas, G., Ernfors, P., Castrén, M., and Castrén, E. (2005). Brain-derived neurotrophic factor and antidepressant drugs have different but coordinated effects on neuronal turnover, proliferation, and survival in the adult dentate gyrus. J. Neurosci. 25, 1089-1094. doi: 10.1523/JNEUROSCI.3741-04.2005

Salehi, A., Delcroix, J. D., and Mobley, W. C. (2003). Traffic at the intersection of neurotrophic factor signaling and neurodegeneration. Trends Neurosci. 26, 73-80. doi: 10.1016/S0166-2236(02)00038-3

Sanchez, M. M., Das, D., Taylor, J. L., Noda, A., Yesavage, J. A., and Salehi, A. (2011). BDNF polymorphism predicts the rate of decline in skilled task performance and hippocampal volume in healthy individuals. Transl. Psychiatry 1:e51. doi: 10.1038/tp.2011.47

Seri, B., Garcia-Verdugo, J. M., Collado-Morente, L., McEwen, B. S., and AlvarezBuylla, A. (2004). Cell types, lineage, and architecture of the germinal zone in the adult dentate gyrus. J. Comp. Neurol. 478, 359-378. doi: 10.1002/cne.20288

Seri, B., Garcia-Verdugo, J. M., McEwen, B. S., and Alvarez-Buylla, A. (2001). Astrocytes give rise to new neurons in the adult mammalian hippocampus. J. Neurosci. 21, 7153-7160.

Spalding, K. L., Bergmann, O., Alkass, K., Bernard, S., Salehpour, M., Huttner, H. B., et al. (2013). Dynamics of hippocampal neurogenesis in adult humans. Cell 153, 1219-1227. doi: 10.1016/j.cell.2013.05.002
Szuhany, K. L., Bugatti, M., and Otto, M. W. (2015). A meta-analytic review of the effects of exercise on brain-derived neurotropic factor. J. Psychiatr. Res. 60, 56-64. doi: 10.1016/j.jpsychires.2014.10.003

Terracciano, A., Martin, B., Ansari, D., Tanaka, T., Ferrucci, L., Maudsley, S., et al. (2010). Plasma BDNF concentration, Val66Met genetic variant and depression- related personality traits. Genes Brain Behav. 9, 512-518. doi: 10.1111/j.1601-183X.2010.00579.x

Trinchero, M. F., Buttner, K. A., Sulkes Cuevas, J. N., Temprana, S. G., Fontanet, P. A., Monzón-Salinas, M. C., et al. (2017). High plasticity of new granule cells in the aging hippocampus. Cell Rep. 21, 1129-1139. doi: 10.1016/j.celrep.2017.09.064

van Praag, H., Kempermann, G., and Gage, F. H. (1999). Running increases cell proliferation and neurogenesis in the adult mouse dentate gyrus. Nat. Neurosci. 2, 266-270. doi: 10.1038/6368

van Praag, H., Shubert, T., Zhao, C., and Gage, F. H. (2005). Exercise enhances learning and hippocampal neurogenesis in aged mice. J. Neurosci. 25, 8680-8685. doi: 10.1523/JNEUROSCI.1731-05.2005

Vaynman, S. S., Ying, Z., Yin, D., and Gomez-Pinilla, F. (2006). Exercise differentially regulates synaptic proteins associated to the function of BDNF. Brain Res. 1070, 124-130. doi: 10.1016/j.brainres.2005.11.062

Vaynman, S., Ying, Z., and Gomez-Pinilla, F. (2004). Hippocampal BDNF mediates the efficacy of exercise on synaptic plasticity and cognition. Eur.J. Neurosci. 20, 2580-2590. doi: 10.1111/j.1460-9568.2004.03720.x

Voss, M. W., Erickson, K. I., Prakash, R. S., Chaddock, L., Kim, J. S., Alves, H., et al. (2013a). Neurobiological markers of exercise-related brain plasticity in older adults. Brain Behav. Immun. 28, 90-99. doi: 10.1016/j.bbi.2012.10.021

Voss, M. W., Heo, S., Prakash, R. S., Erickson, K. I., Alves, H., Chaddock, L., et al. (2013b). The influence of aerobic fitness on cerebral white matter integrity and cognitive function in older adults: results of a one-year exercise intervention. Hum. Brain Mapp. 34, 2972-2985. doi: 10.1002/hbm.22119

Wang, J., Yang, L., Zhang, Y., Niu, C., Cui, Y., Feng, W., et al. (2015). BDNF and COX-2 participate in anti-depressive mechanisms of catalpol in rats undergoing chronic unpredictable mild stress. Physiol. Behav. 151, 360-368. doi: 10.1016/j.physbeh.2015.08.008

Waterhouse, E. G., An, J. J., Orefice, L. L., Baydyuk, M., Liao, G., Zheng, K., et al. (2012). BDNF promotes differentiation and maturation of adultborn neurons through GABAergic transmission. J. Neurosci. 32, 14318-14330. doi: 10.1523/JNEUROSCI.0709-12.2012

Weissman, I. L., Anderson, D. J., and Gage, F. (2001). Stem and progenitor cells: origins, phenotypes, lineage commitments, and transdifferentiations. Annu. Rev. Cell Dev. Biol. 17, 387-403. doi: 10.1146/annurev.cellbio.17.1.387

Wrann, C. D., White, J. P., Salogiannis, J., Laznik-Bogoslavski, D., Wu, J., Ma, D., et al. (2013). Exercise induces hippocampal BDNF through a PGC$1 \alpha /$ FNDC5 pathway. Cell Metab. 18, 649-659. doi: 10.1016/j.cmet.2013. 09.008

Yoshii, A., and Constantine-Paton, M. (2010). Post-synaptic BDNF-TrkB signaling in synapse maturation, plasticity and disease. Dev. Neurobiol. 70, 304-322. doi: 10.1002/dneu.20765

Zuccato, C., and Cattaneo, E. (2009). Brain-derived neurotrophic factor in neurodegenerative diseases. Nat. Rev. Neurol. 5, 311-322. doi: $10.1038 /$ nrneurol.2009.54

Conflict of Interest Statement: The authors declare that the research was conducted in the absence of any commercial or financial relationships that could be construed as a potential conflict of interest.

Copyright (c) 2018 Liu and Nusslock. This is an open-access article distributed under the terms of the Creative Commons Attribution License (CC BY). The use, distribution or reproduction in other forums is permitted, provided the original author(s) and the copyright owner are credited and that the original publication in this journal is cited, in accordance with accepted academic practice. No use, distribution or reproduction is permitted which does not comply with these terms. 